\title{
INTERFACIAL ELEMENT FOR FINITE ELEMENT MODAL ANALYSIS OF BOLTED JOINTS
}

\section{YOSHINAO KISHIMOTO ${ }^{1}$, YUKIYOSHI KOBAYASHI ${ }^{1}$, TOSHIHISA OHTSUKA ${ }^{1}$, TAKUYA SHINOHARA ${ }^{1}$ AND NAOKI JIMBO'}

\author{
${ }^{1}$ Department of Mechanical Engineering, Tokyo City University \\ 1-28-1 Tamazutsumi, Setagaya-ku, Tokyo, Japan \\ ykishimo@tcu.ac.jp, http://www.tcu.ac.jp
}

Key words: Finite Element Method, Multi-scale Approach, Interfacial Stiffness.

\begin{abstract}
Multi-material structures are going to be a main scheme to construct automobiles. For the construction of multi-material structures, techniques to join dissimilar materials are required. The major joining techniques are classified into welding, adhesion and mechanical fastening such as bolted joints and riveting. Especially, bolted joints enable joining of metallic materials (steel and aluminium alloy, etc.) and non-metallic materials (CFRP, etc.) with high joint strength. However, the total stiffness of structures with bolted joints is relatively low because interfaces in bolted joints just contact each other, and its interfacial stiffness is lower than elastic modulus of base materials. Moreover, interfacial stiffness of bolted joints depends on clamping force of bolt and nut. This study has proposed an interfacial element for finite element modal analysis of bolted joints. The interfacial element simulates interfacial stiffness of bolted joints. Contact of interfaces is assumed to be the Hertzian contact of elastic asperities whose peak heights obey the Gaussian distribution. Based on this assumption, the stiffness of the interfacial element is derived from the compressive stress and the surface texture of the interfaces. By using the finite element model with the interfacial element, the modal analysis computes the natural frequency and the vibration mode. Finite element simulations and hammering tests have been conducted with several bolted joints. In general, the natural frequency of the bolted joints in the hammering tests increases with the increase in the clamping force, but it is lower than the calculation results in which the stiffness reduction of the jointed interfaces is ignored. The calculation results by using the proposed interfacial element agree with the hammering tests. Therefore, the proposed interfacial element contributes to improvement of modal analysis of bolted joints by mathematically modelling stiffness reduction of jointed interfaces based on tribology.
\end{abstract}

\section{INTRODUCTION}

Multi-material structures are going to be a main scheme to construct automobiles. For the construction of multi-material structures, techniques to join dissimilar materials are required. The major joining techniques are classified into welding, adhesion and mechanical fastening such as bolted joints and riveting. Especially, bolted joints enable joining of metallic materials (steel and aluminium alloy, etc.) and non-metallic materials (CFRP, etc.) with high joint 
strength. In order to achieve further weight saving, bolted joints are sometimes applied to strengthen adhesion whose joint strength is much lower than bolted joints [1-4].

However, the total stiffness of structures with bolted joints is lower than that of welding parts because interfaces in bolted joints just contact each other, and its interfacial stiffness is lower than elastic modulus of base materials. Moreover, interfacial stiffness of bolted joints depends on clamping force of bolt and nut. The estimation of the interfacial stiffness is one of the most important concerns for the design of structures in that interfaces of joint parts are often the weakest part in the structure. In the field of tribology, contact mechanics and mathematical models of micro asperities have been discussed [5-10]. The general approach seems to assume that the peak heights of the asperities obey the Gaussian distribution and each contact of the asperities is the Hertzian contact. With the development of computational approaches, some studies in recent years have utilized the finite element method (FEM) for the contact analysis of the interfaces in microscale [11-14]. These studies have measured or preliminarily presumed the surface profile of the interfaces and created the finite element (FE) model of the micro asperities. Although the contact analysis by using the surface profile of the interfaces in microscale is one of the accurate estimations, the contact analysis takes large computation cost if macroscale phenomena such as vibration of structures are simulated.

This study has proposed an interfacial element for finite element modal analysis of bolted joints. The interfacial element simulates interfacial stiffness of bolted joints. Contact of interfaces is assumed to be the Hertzian contact of elastic asperities whose peak heights obey the Gaussian distribution. Based on this assumption, the stiffness of the interfacial element is derived from the conn finite element model wi frequency and the vibration The previous paper simplified case that thread surfaces and seating

in specimens [15]. However, effect of thread surfaces and seating surfaces on the natural

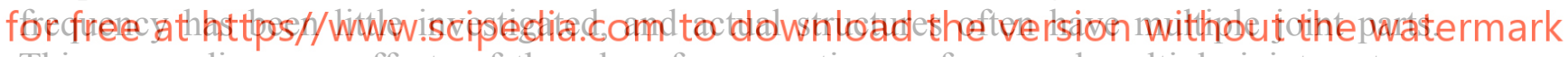
This paper discusses effects of thread surfaces, seating surfaces and multiple joint parts as further study. In order to investigate these details, finite element simulations and hammering tests have been conducted with several bolted joints.

\section{INTERFACIAL ELEMENT [15]}

Figure 1 shows the contact model of nominally flat surfaces. As shown in the left of Fig. 1, the pieces 1 and 2 are joined by the compressive stress $p_{z} \cdot z$-axis is defined along the normal direction of the interfaces of the pieces. $x$-axis and $y$-axis are defined to be normal to each other as shown in Fig. 1. The contact model describes the contacts of the spherical asperities shown in the middle of Fig. 1. The orthotropic model shown in the right of Fig. 1 simulates the interfacial stiffness. The total height of the asperities is $L$, and the gap between the mean planes of the asperity peak heights is $d$. The elastic modulus $E_{z}, G_{y z}$ and $G_{z x}$ are derived by the following process, and the elastic modulus on the other directions is set to zero.

Assuming that the asperity peak heights obey the Gaussian distribution and the contact of asperities is the Hertzian contact, the relationship between the nominal compressive stress $p_{z}$ 


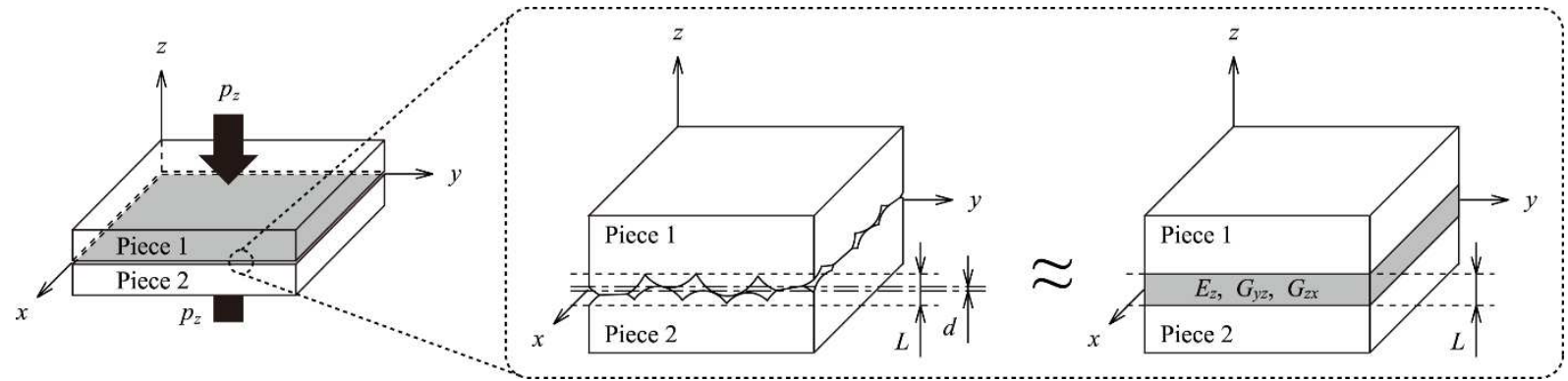

Figure 1: Contact model of nominally flat surfaces

and the gap $d$ is described by using the homogenized elastic modulus $C$ as follows [6].

$$
p_{z}=C \cdot F_{5 / 2}(d)
$$

where

$$
\begin{gathered}
F_{m}(d)=\frac{1}{\sqrt{2 \pi}} \int_{0}^{\infty} \zeta^{m} \exp \left[-\frac{1}{2}\left(\zeta+\frac{d}{\sigma}\right)^{2}\right] d \zeta \\
\sigma=\sqrt{\sigma_{1}{ }^{2}+\sigma_{2}{ }^{2}}
\end{gathered}
$$

Using the subscript $i$ as the identifier for the piece $i=1,2, \sigma_{i}$ is the standard deviation of the asperity peak heights

follows.
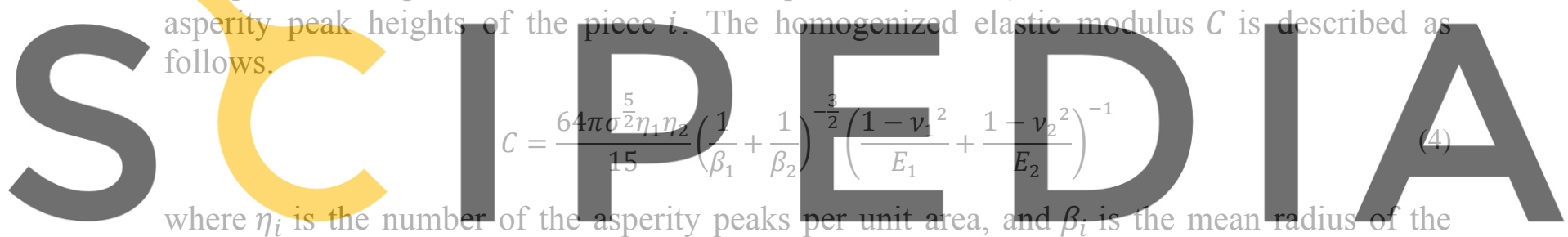

curvature at asperity peaks. $E_{i}$ and $v_{i}$ are the Young's modulus and the Poisson's ratio of the

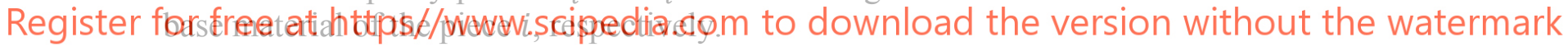

Assuming that the small displacement occurs in the vibration of the specimen, the $z$ direction Young's modulus of the interfaces can be described as follows.

$$
E_{z}=-\frac{\partial p_{z}}{\partial(d / L)}=\left(\frac{F_{7 / 2}(d)}{F_{5 / 2}(d)}+\frac{d}{\sigma}\right) \frac{p_{z} \cdot L}{\sigma}
$$

Similarly, Björklund derived the relationship between the shear stress and the displacement tangential to the interfaces under the compressive stress $p_{z}$ based on the Mindlin's theory [8]. Assuming that the stiffness in the tangential direction to the interfaces is isotropic, the shear modulus is described as follows.

$$
G_{y z}=G_{z x}=4\left(\frac{1-v_{1}^{2}}{E_{1}}+\frac{1-v_{2}^{2}}{E_{2}}\right)\left(\frac{2-v_{1}}{G_{1}}+\frac{2-v_{2}}{G_{2}}\right)^{-1} E_{z}
$$

where $G_{y z}$ is the shear modulus at the $y$-surface in the $z$-direction, and $G_{z x}$ is the shear modulus at the $x$-surface in the $z$-direction. $G_{i}(i=1,2)$ is the shear modulus of the base material of the piece $i$. In the FEM using the above model, the finite element whose elastic modulus are given by Eq. (5) and Eq. (6) is inserted into the interfaces. The finite element is named as the interfacial element, hereinafter. 


\section{EXPERIMENT}

\subsection{Specimen and FE model}

This study has prepared two types of bolted joints: single bolted joint and L-shaped bolted joint. Figure 2 shows components of the single bolted joint. The single bolted joint is composed of a set of M36 bolt, M36 nut and collar. All of them are made of steel (Young's modulus $E_{i}=210 \mathrm{GPa}$, Poisson's ratio $v_{i}=0.3$, mass density $\rho_{i}=7860 \mathrm{~kg} / \mathrm{m}^{3}$ ). Figure 3 shows FE model of the single bolted joint. The FE model is composed of 8 nodes hexahedral elements. The total numbers of nodes and elements are 9190 and 6687, respectively.

Figure 4 shows schematic of the L-shaped bolted joint. The L-shaped bolted joint is composed of two aluminum plates and an aluminum bracket (Young's modulus $E_{i}=68.3$ GPa, Poisson's ratio $v_{i}=0.34$, mass density $\rho_{i}=2700 \mathrm{~kg} / \mathrm{m}^{3}$ ) fasten by 15 sets of M6 steel bolt and nut (Young's modulus $E_{i}=210 \mathrm{GPa}$, Poisson's ratio $v_{i}=0.3$, mass density $\rho_{i}=$ $7860 \mathrm{~kg} / \mathrm{m}^{3}$ ). Figure 5 shows FE model of the L-shaped bolted joint. Taking account of the symmetry, the FE mesh is applied on a half of the L-shaped bolted joint and the FE model is also composed of 8 nodes hexahedral elements. The total numbers of nodes and elements are 13937 and 9503, respectively.
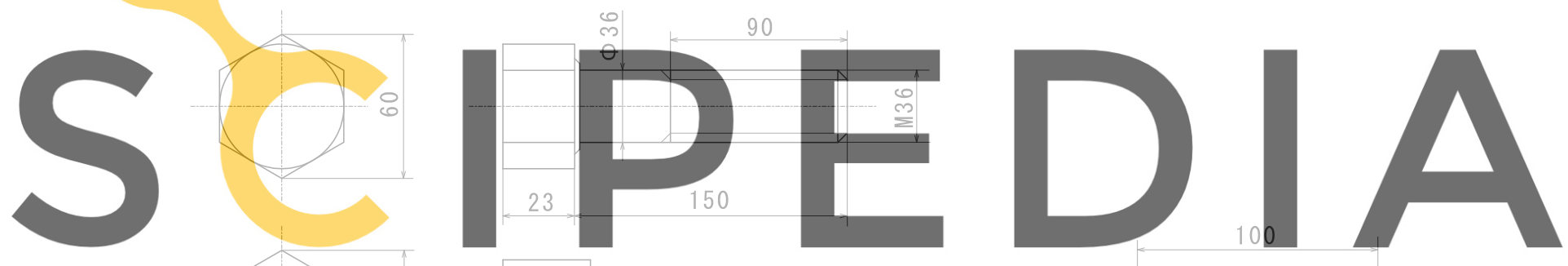

Register for free at https//www.scipediå.com to download th
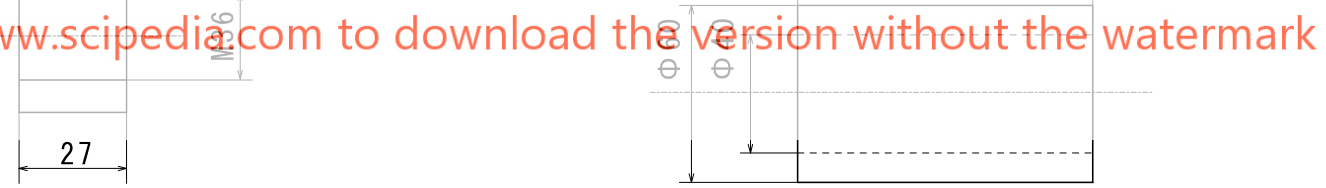

Figure 2: Components of single bolted joint
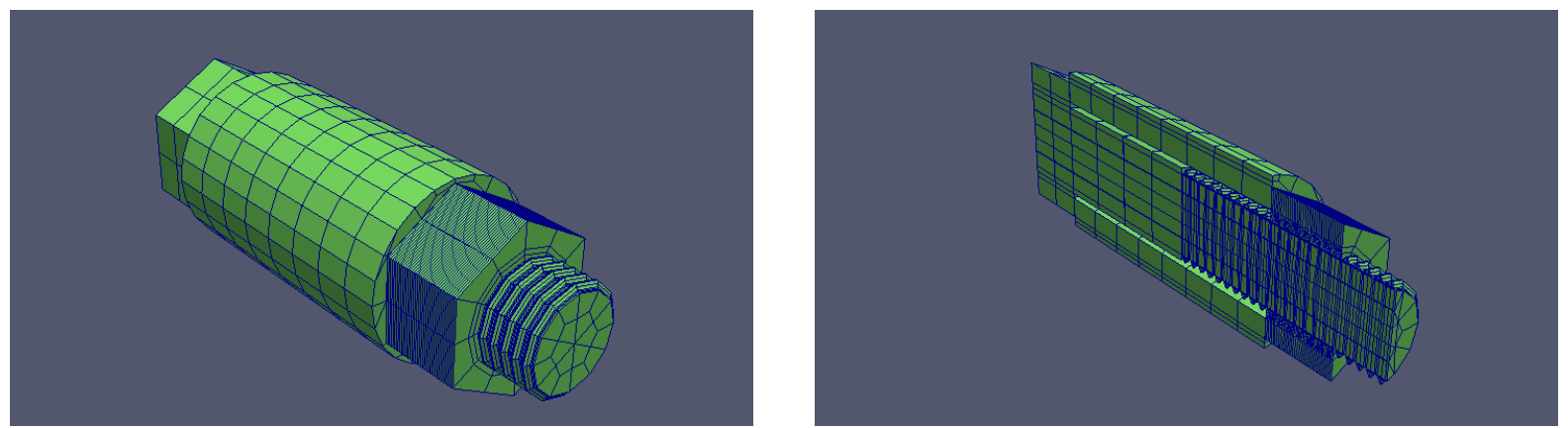

Figure 3: FE model of single bolted joint 
Figure 6 shows interfacial elements in the FE models. The interfacial elements are inserted into all of the interfaces in the single bolted joint, and the interfaces between the plates and the bracket in the L-shaped bolted joint. The thread surfaces and the seating surfaces of the Lshaped bolted joint are ignored by taking into account the result of the single bolted joint described later.
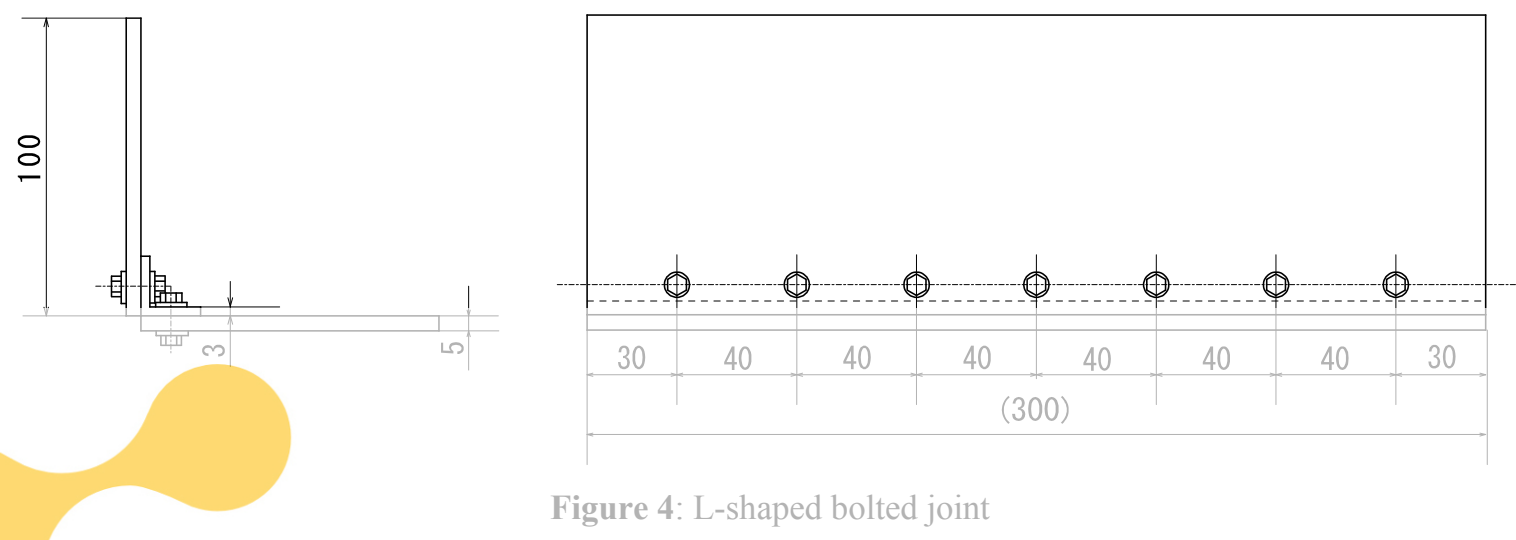

Figure 4: L-shaped bolted joint

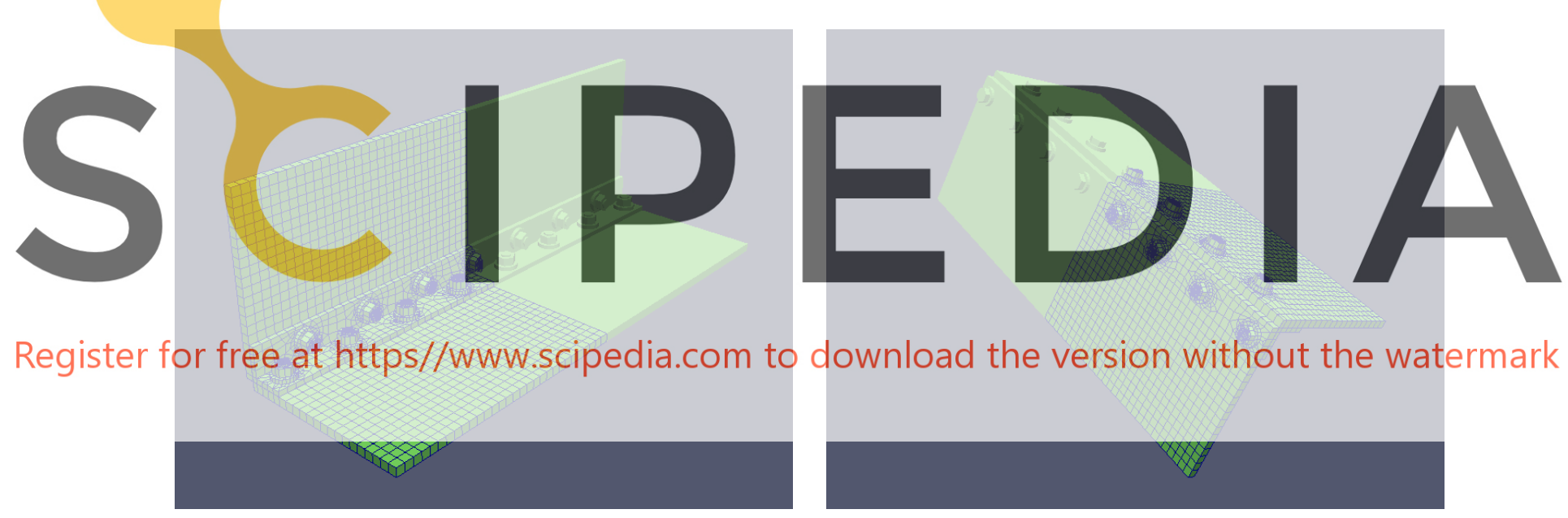

Figure 5: FE model of L-shaped bolted joint
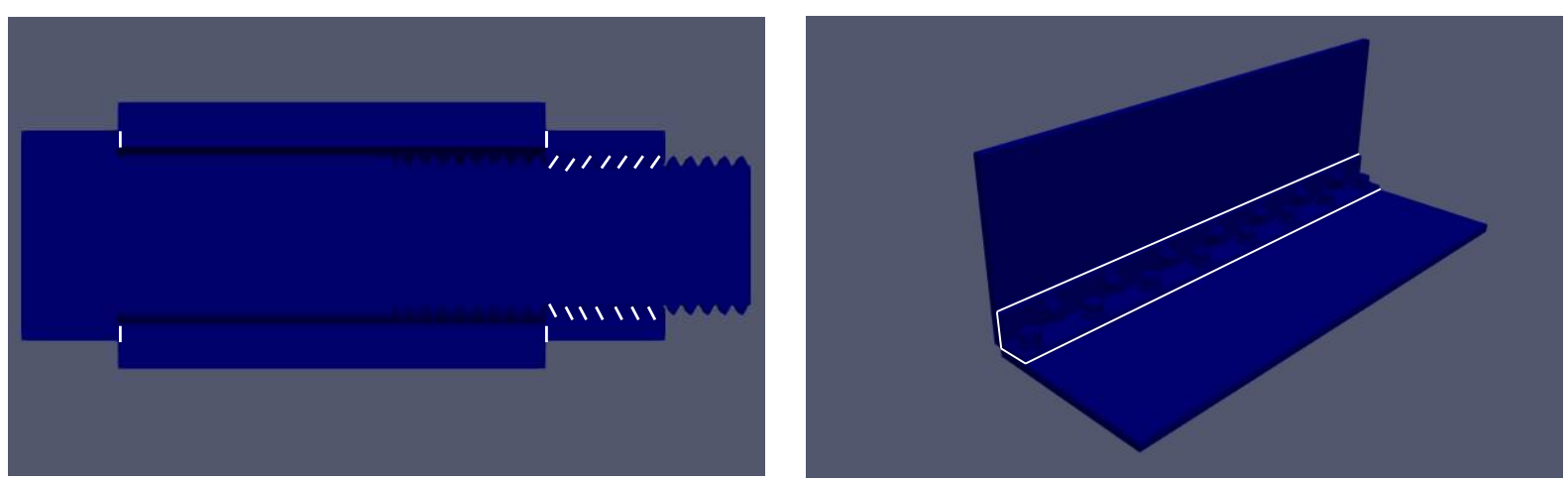

Figure 6: Interfacial elements (white lines) in FE models 


\subsection{Test procedure}

Figure 7 shows pictures of the experimental setups. Surface profiles of the interfaces of the bolted joints were measured by the surface roughness meter (SJ-411 made by Mitutoyo Corporation) before assembling and after disassembling the bolted joints. The surface profiles were not changed by the assembling. Table 1 shows the surface texture parameters derived by the surface profiles.

In the hammering test, the bolted joints were put on soft sponges, and two acceleration pickups (NP-3211 made by Ono Sokki Co., Ltd) were attached to the bolted joints. The clamping force of the bolts and nuts were measured by the pressure measurement films (Prescale made by Fujifilm Corporation) inserted into the bolted joints. After the clamping force was applied by the torque wrench, the bolted joints were hammered by the impulse hammer (GK-3100 made by Ono Sokki Co., Ltd) and the acceleration response was measured by the acceleration pickups. In this test, all of the sets of the bolts and nuts were tightened by the same tightening torque. The natural frequency of the bolted joints was obtained from Fourier spectra of the acceleration response measured by the acceleration pickups.

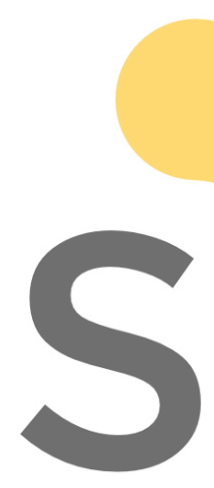

\section{Pressure measurement film}

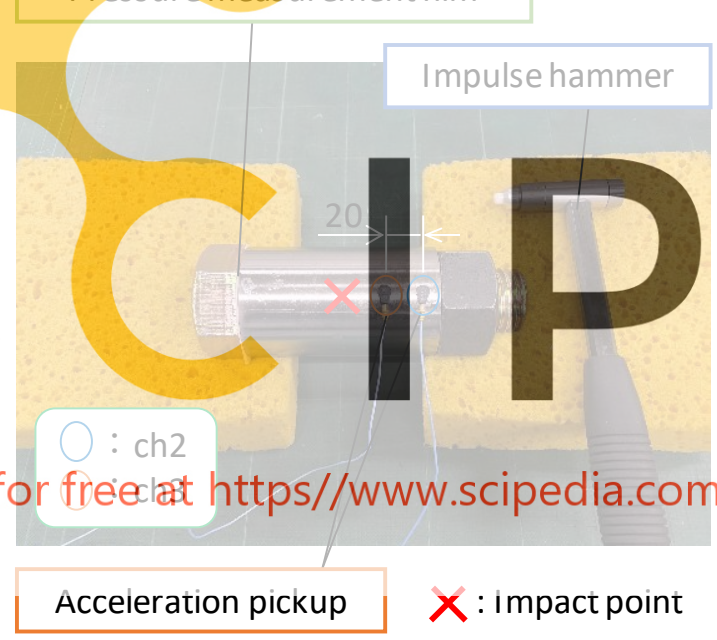

(a) Single bolted joint

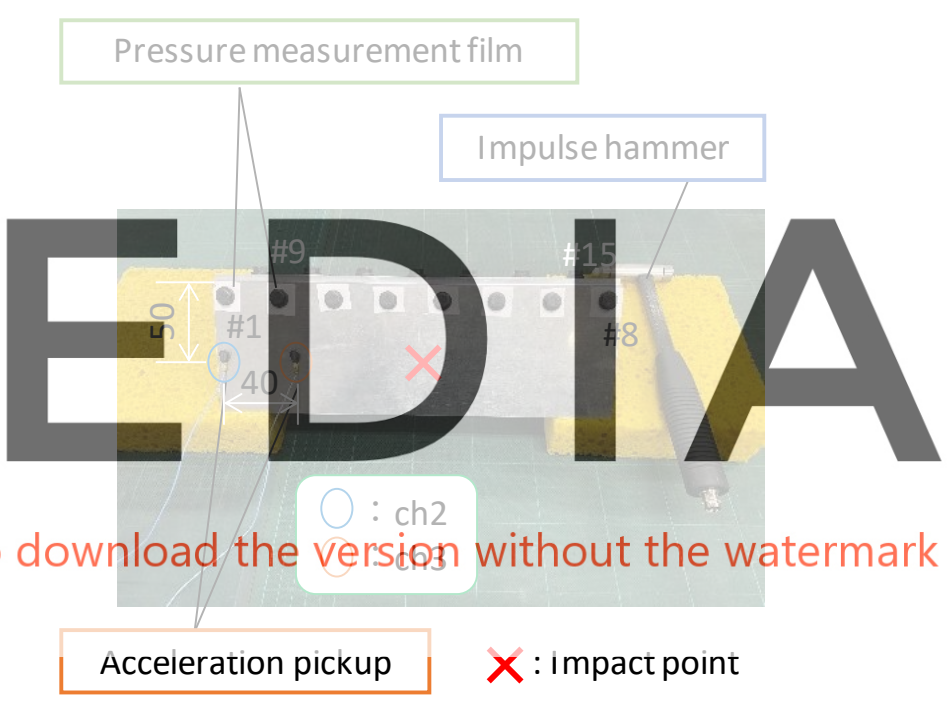

(b) L-shaped bolted joint

Figure 7: Experimental setup

Table 1: Surface texture parameters of bolted joints

\begin{tabular}{cccc}
\hline \multirow{2}{*}{$\begin{array}{c}\text { Bolted } \\
\text { joints }\end{array}$} & Interface & $\begin{array}{c}\text { Standard deviation of } \\
\text { asperity peak heights } \\
\sigma[\mu \mathrm{m}]\end{array}$ & $\begin{array}{c}\text { Homogenized } \\
\text { elastic modulus } \\
C[\mathrm{GPa}]\end{array}$ \\
\hline \multirow{2}{*}{$\begin{array}{c}\text { Single } \\
\text { bolted joint }\end{array}$} & Thread surfaces (bolt and nut) & 3.11 & 40.2 \\
\cline { 2 - 4 } & Seating surface (bolt and collar) & 1.24 & 1.76 \\
\cline { 2 - 4 } & Seating surface (nut and collar) & 3.27 & 11.4 \\
\hline \multirow{2}{*}{$\begin{array}{c}\text { L-shaped } \\
\text { bolted joint }\end{array}$} & Plate and bracket (with 7 holes) & 0.389 & 0.188 \\
\cline { 2 - 4 } & Plate and bracket (with 8 holes) & 0.491 & 0.461 \\
\hline
\end{tabular}




\section{RESULTS AND DISCUSSION}

Figure 8 shows the Fourier spectra in the hammering test and the vibration modes in the FE simulation of the single bolted joint. Three major peaks appeared under $20 \mathrm{kHz}$ in the Fourier spectra as shown as the black lines (b)-(d). The frequencies at the peaks are the natural frequency, and these vibration modes in the FE simulation are (b) 1st bending mode, (c) 1 st torsional mode and (d) 2nd bending mode, respectively. Figure 9 shows the natural frequencies in the hammering test and the FE simulation. In Fig. 9, the dashed curves (proposed) indicate the calculation results of the FE models applied with the interfacial elements shown in Fig. 6. The solid lines (conventional) indicate the calculation results when the effect of the interfacial stiffness is ignored. Specifically, the material constants of the interfacial elements are set to those of the base material (steel, Young's modulus $E_{i}=210$ GPa, Poisson's ratio $v_{i}=0.3$, mass density $\rho_{i}=7860 \mathrm{~kg} / \mathrm{m}^{3}$ ) in order to obtain the solid lines. As shown in Fig. 9, the calculation results of the FE model with the interfacial elements increase with the increase in the clamping force and come close to the calculation results in which the effect of the interfacial stiffness is ignored under enough clamping force. The natural frequency in the hammering test has in good agreement with the calculation results. Therefore, the stiffness reduction seems to occur, but the stiffness reduction of the interfaces at the thread surfaces and the seating surfaces could be ignored under enough clamping force.

Figure 10 shows the Fourier spectra in the hammering test and the vibration modes in the FE simulation of the L-shaped bolted joint. As shown in Fig. 10 (a), there are a number of

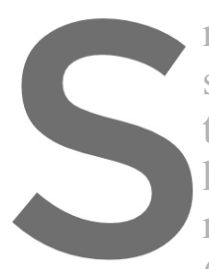
major peaks under symmetric vibration the vibration modes hammering test and the results of the EE mode (conventional) indicate the calculation results
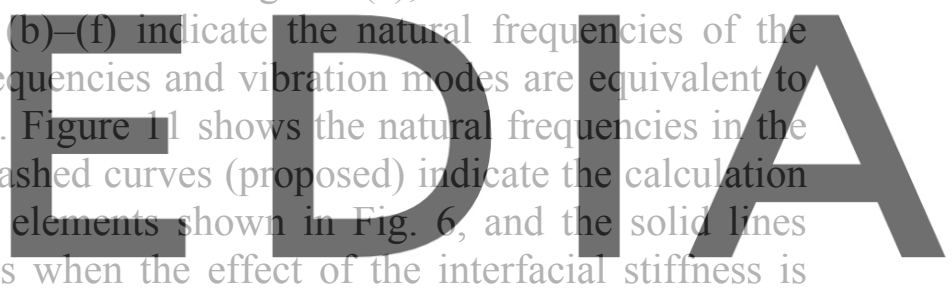
ignored as with Fig. 9. As shown in Fig. 11, the natural frequency in the hammering test

the interfacial elements agree with the results of the hammering test at all of the vibration modes. Mioreover, the interfacial stiffness of the thread surfaces and the seating surfaces of the L-shaped bolted joint seems to can be ignored. On the other hand, the calculation results in which the effect of the interfacial stiffness is ignored is higher than the results of the hammering test and the FE simulation by using the interfacial elements. Therefore, also in the L-shaped bolted joint, the stiffness reduction of the jointed interfaces occurs, and the proposed interfacial element enables the improvement of the vibration simulation.

\section{CONCLUSIONS}

This study has proposed an interfacial element for finite element modal analysis of bolted joints and conducted finite element simulations and hammering tests with several bolted joints. The calculation results by using the proposed interfacial element agree with the hammering tests, and the interfacial stiffness at the thread surfaces and the seating surfaces seems to can be ignored. The proposed interfacial element contributes to improvement of modal analysis of bolted joints by mathematically modelling stiffness reduction of jointed interfaces based on tribology. This work was supported by JSPS KAKENHI Grant Number JP18K03849. 


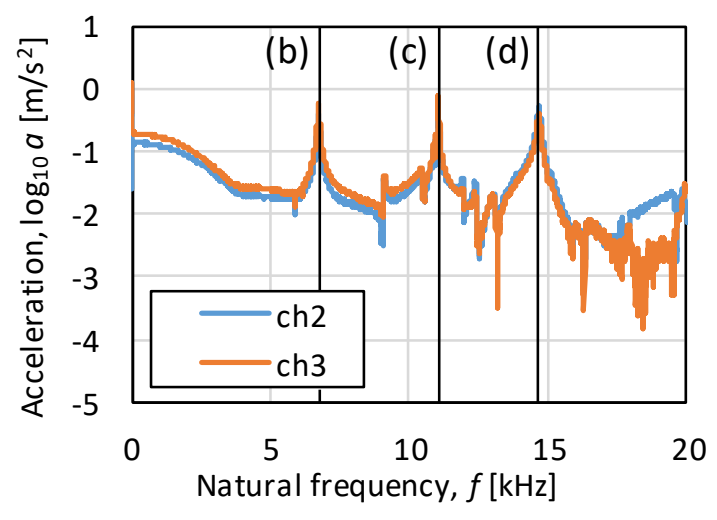

(a) Fourier spectra at clamping force $6 \mathrm{kN}$

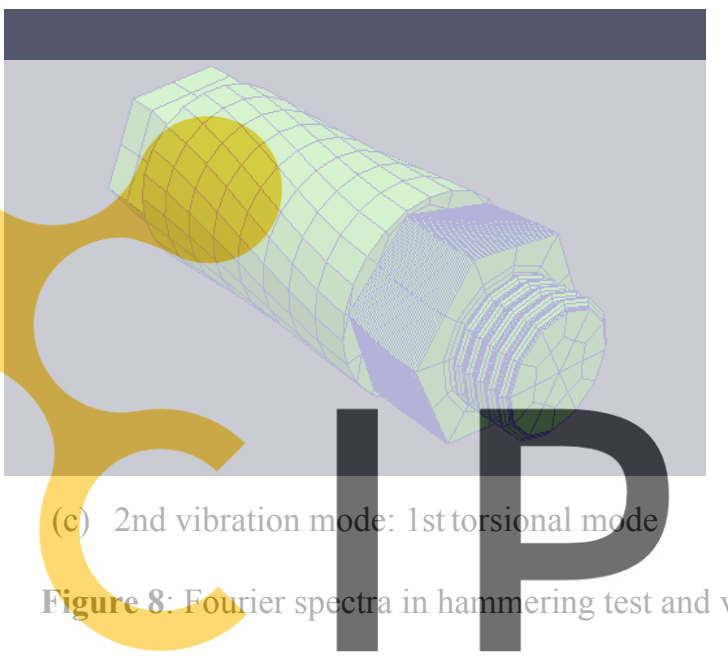

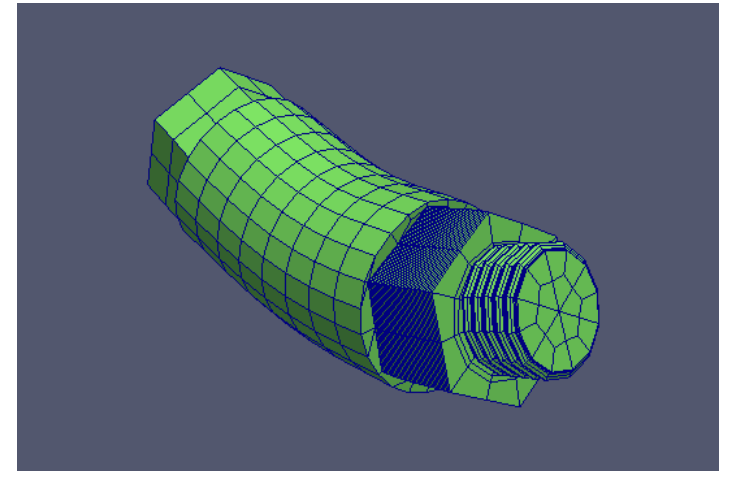

(b) 1 st vibration mode: 1 st bending mode

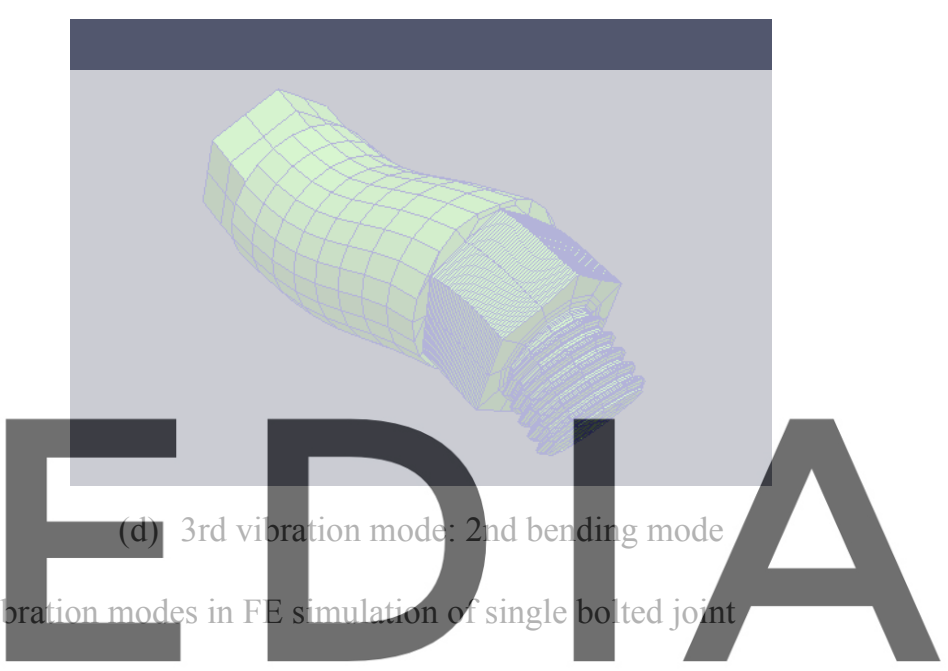

Register for fregoot https//www.scipedia.com to download the version without the watermark

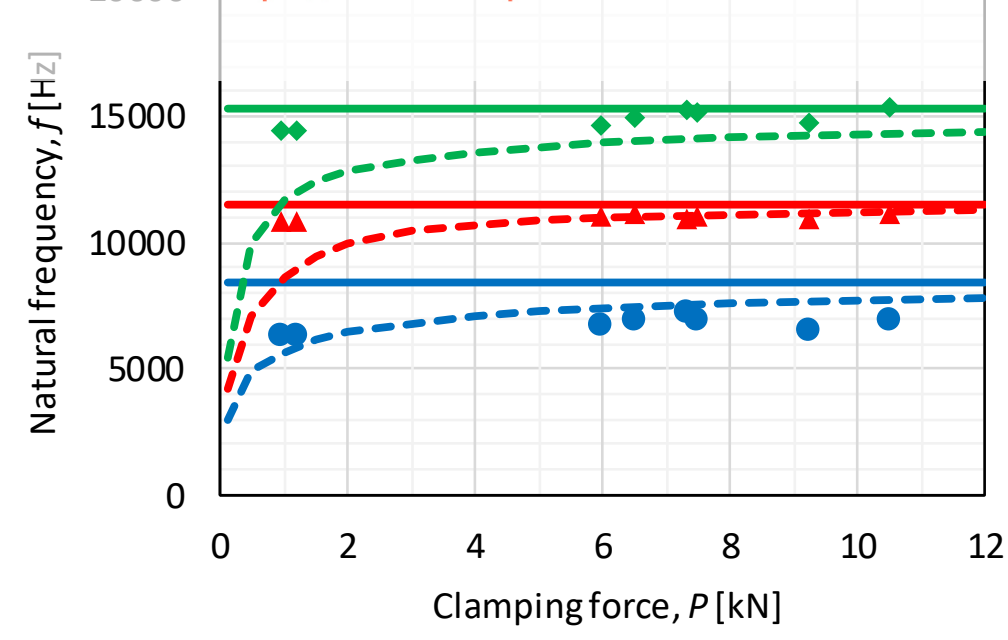

- 1st, num. proposed

1st, num. conventional

- 2nd, hammering test

- 2nd, num. proposed

2nd, num. conventional

- 3rd, hammering test

- 3rd, num. proposed

3rd, num. conventional

Figure 9: Natural frequency of single bolted joint 


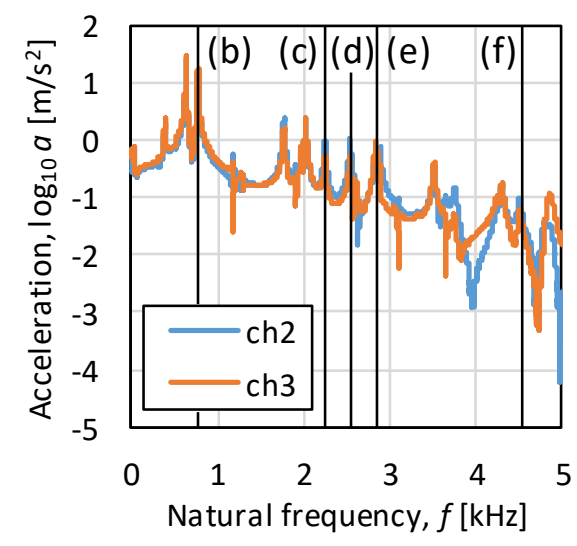

(a) Fourier spectra at clamping force $2 \mathrm{kN}$

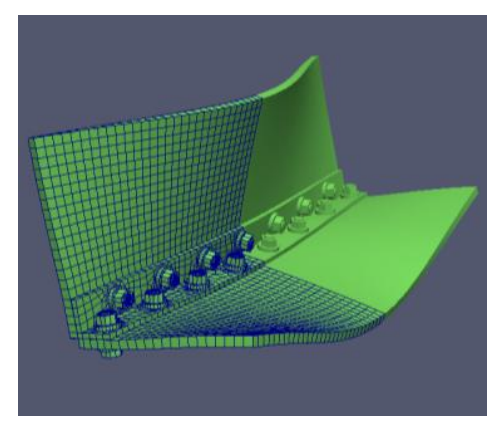

(b) 1st vibration mode

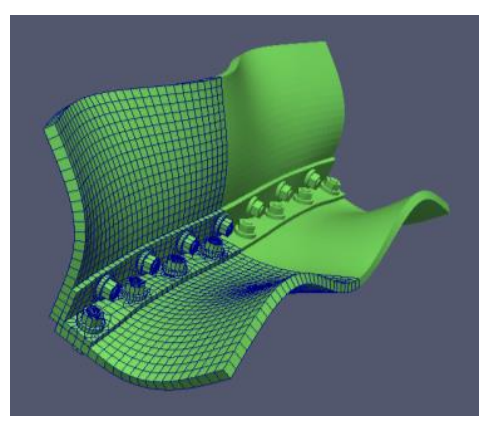

(c) 2nd vibration mode
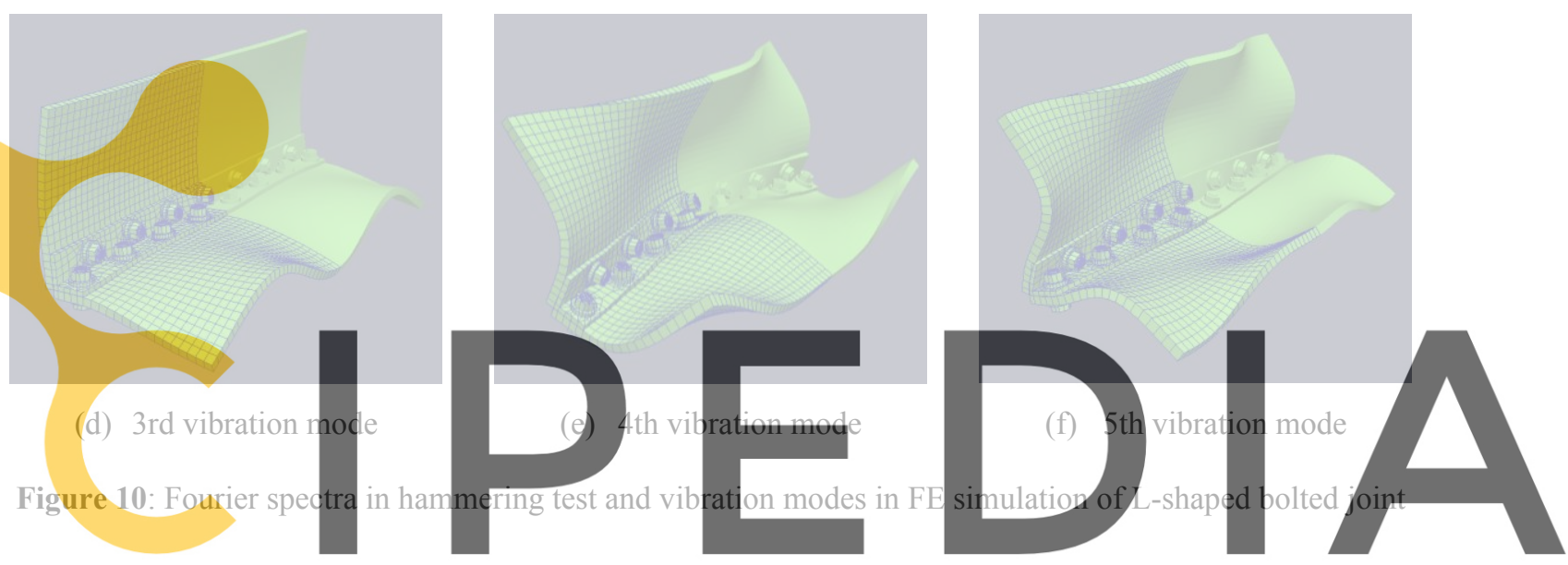

5000

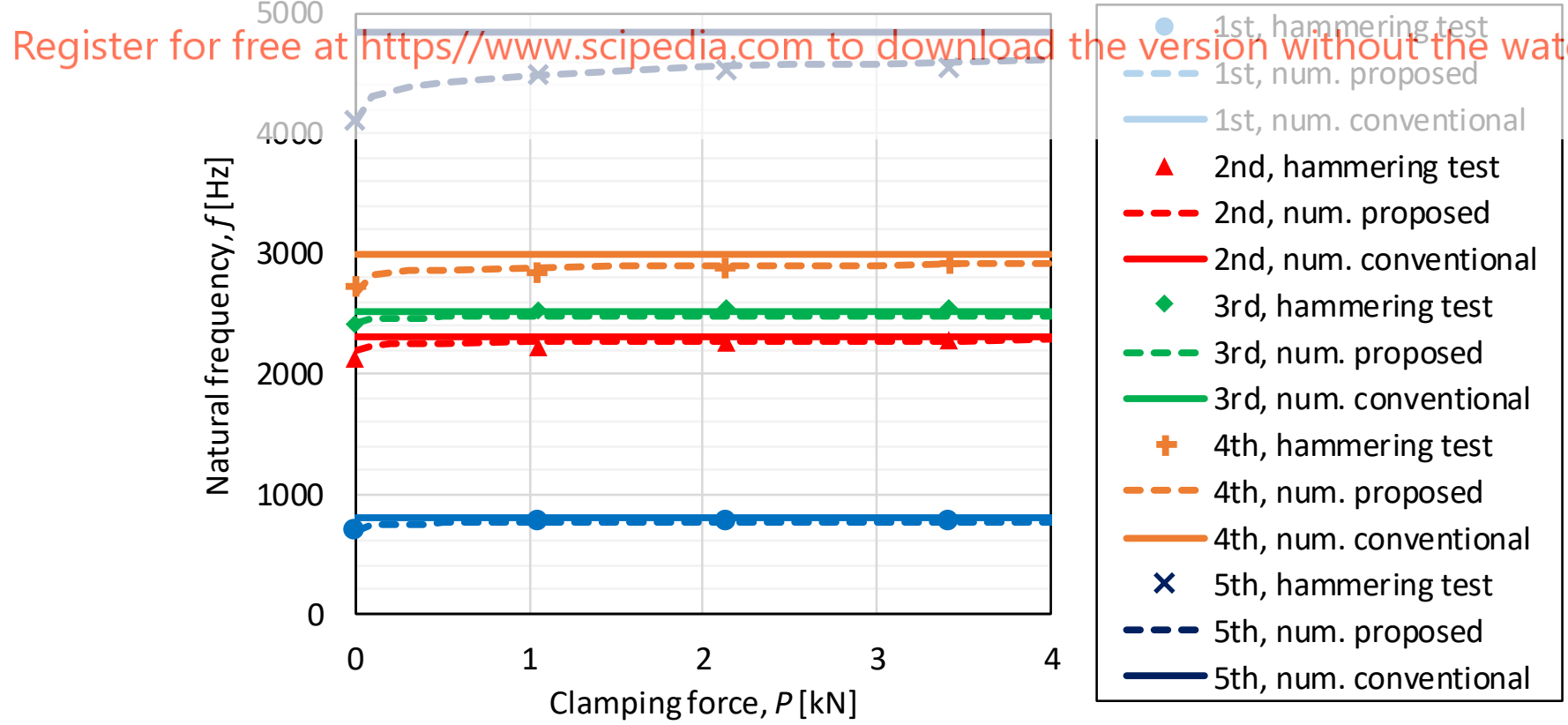

Figure 11: Natural frequency of L-shaped bolted joint 


\section{REFERENCES}

[1] Nichols, J. M., Trickey, S. T., Seaver, M., Motley, S. R. and Eisner, E. D., Using ambient vibrations to detect loosening of a composite-to-metal bolted joint in the presence of strong temperature fluctuations, Transactions of the ASME, Journal of Vibration and Acoustics (2006) 129 (6):710-717.

[2] Catalanotti, G., Camanho, P. P., Ghys, P. and Marques, A. T., An efficient design method for multi-material bolted joints used in the railway industry, Composite Structures (2011) 94 (1):246-252.

[3] Coelho, A. M. G. and Mottram, J. T., A review of the behaviour and analysis of bolted connections and joints in pultruded fibre reinforced polymers, Materials \& Design (2015) 74:86-107.

[4] Hammami, C., Balmes, E. and Guskov, M., Numerical design and test on an assembled structure of a bolted joint with viscoelastic damping, Mechanical Systems and Signal Processing (2016) 70-71:714-724.

[5] Greenwood, J. A. and Williamson, J. B. P., Contact of nominally flat surfaces, Proceedings of the Royal Society A, Mathematical, Physical and Engineering Sciences (1966) 295 (1442):300-319.

[6] Greenwood, J. A. and Tripp, J. H., The contact of two nominally flat rough surfaces, Proceedings of the Institution of Mechanical Engineers (1970) 185:625-633.

[77 Olofsson, U., Cyclic micro-slip under unlubricated conditions, Tribology International (1995) 28 (4):207-2

[8] Björklund, S., Transactions of the

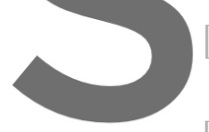
] Olofsson, U. and Hagmai deformation of ellipsoidal Hagman, L. A. and Olofsson, U., A modi
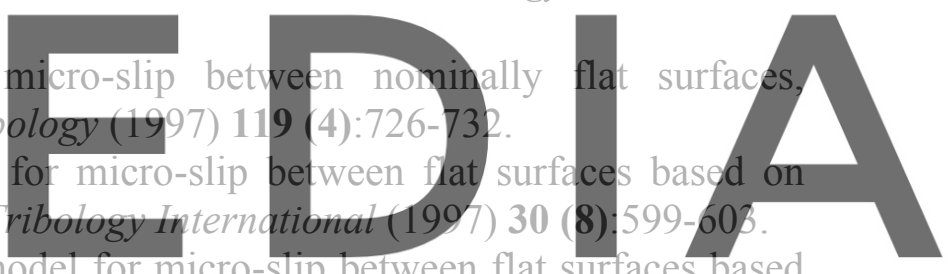
on deformation of ellipsoidal elastic asperities: parametric study and experimental

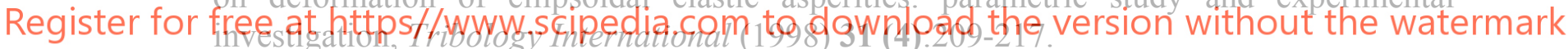

[11] Karupannasamy, D. K., Rooij, M. B. and Schipper, D. J., Multi-scale friction modeiling for rough contacts under siiding conditions, Wear (2013) 308:222-231.

[12] Hol, J., Meinders, V. T., Rooij, M. B. and Boogaard, A. H., Multi-scale friction modeling for sheet metal forming: The boundary lubrication regime, Tribology International (2015) 81:112-128.

[13] Wagner, P., Wriggers, P., Klapproth, C., Prange, C. and Wies, B., Multiscale FEM approach for hysteresis friction of rubber on rough surfaces, Computer Methods in Applied Mechanics and Engineering (2015) 296:150-168.

[14] Santhapuram, R. R. and Nair, A. K., Frictional properties of multi-asperity surfaces at the nanoscale, Computational Materials Science (2017) 136:253-263.

[15] Kishimoto, Y., Kobayashi, Y., Ohtsuka, T., Matsumoto, A. and Niizuma, M., Estimation method of interfacial stiffness of bolted joint in multi-material structure by inverse analysis, Mechanical Engineering Journal (2019) 6 (3):DOI: 10.1299/mej.1800471. 